Cahiers $d u$ MONDE RUSSE

\section{Cahiers du monde russe}

Russie - Empire russe - Union soviétique et États indépendants

$59 / 4 \mid 2018$

Varia

\title{
Serhii Plokhy, ed., The Future of the Past
}

New Perspectives on Ukrainian History

\section{Thomas Chopard}

\section{(2) OpenEdition \\ 1 Journals}

Édition électronique

URL : https://journals.openedition.org/monderusse/10925

DOI : $10.4000 /$ monderusse. 10925

ISSN : $1777-5388$

Éditeur

Éditions de l'EHESS

\section{Édition imprimée}

Date de publication : 1 octobre 2018

Pagination : 721-723

ISBN : 978-2-7132-2747-9

ISSN : 1252-6576

\section{Référence électronique}

Thomas Chopard, «Serhii Plokhy, ed., The Future of the Past », Cahiers du monde russe [En ligne], 59/4 I 2018, mis en ligne le 01 octobre 2018, consulté le 08 janvier 2022. URL : http://

journals.openedition.org/monderusse/10925; DOI : https://doi.org/10.4000/monderusse.10925 


$\begin{array}{cccccccccccccccc}\text { E } & \text { U } & \text { R } & \text { O } & \text { P } & \text { E } & & \text { C } & \text { E } & \text { N } & \text { T } & \text { R } & \text { A } & \text { L } & \text { E } \\ & \text { E } & \text { T } & & \text { O } & \text { R } & \text { I } & \text { E } & \text { N } & \text { T } & \text { A } & \text { L } & \text { E } & & \end{array}$

Serhii PLOKHY, ed.

The Future of the Past

New Perspectives on Ukrainian History

Havard Ukrainian Studies, 34, 1-4 (2015-2016), 552 p.

L'imposant volume 34, paru en 2017, des Harvard Ukrainian Studies, l'une des principales revues anglophones en histoire de l'Ukraine, adossée au centre d'études ukrainiennes de Harvard, rassemble 21 contributions qui tentent toutes à leur manière de faire le point sur les évolutions récentes de l'historiographie. Seuls deux contributeurs enseignent et travaillent en Ukraine (Georgiy Kasianov et Oleksii Tolochko) et un seul en Europe occidentale (Andrea Graziosi). Le point est donc essentiellement focalisé sur l'historiographie produite en Amérique du Nord, dont il faut toutefois souligner les liens entretenus de longue date avec les institutions de recherche et d'enseignement en Ukraine. 
Les sujets des différents articles sont divers, voire hétérogènes, mais visent tous à analyser ou exemplifier un sujet historiographique et reflètent trois conférences qui se sont déroulées en 2012 et 2013. Dans l'ensemble, il s'agit donc plutôt d'un effort de conceptualisation et de catégorisation que de la présentation de nouveaux chantiers de recherche à proprement parler. La première moitié du $\mathrm{XX}^{\mathrm{e}}$ siècle domine sans pour autant écraser les autres périodes. Toutefois, dans ces études de cas et ces essais historiographiques, quelques thèmes prévalent : l'histoire culturelle et intellectuelle, d'une part, l'histoire des guerres et des violences de masse d'autre part. Le volume a notamment l'avantage de résumer sans les escamoter des travaux riches et récents. Les textes de George O. Liber et de Faith Hillis présentent par exemple clairement leurs monographies publiées depuis ${ }^{1}$.

Mark von Hagen posait en 1995 la question de savoir si l'Ukraine avait une histoire en propre et répondait, en dépit d'une sympathie évidente pour son sujet, par la négative ${ }^{2}$. Les études ukrainiennes reflétaient la situation de cet état récent, tiraillé dans son écriture de l'histoire aussi bien que dans son narratif dominant entre différentes approches et différents héritages (impériaux russe et austro-hongrois, soviétique, nationaliste ukrainien, présences des minorités, etc.). Les premières contributions du volume des Harvard Ukrainian Studies tentent donc de faire le point, près de vingt ans plus tard, sur les approches dominantes et nouvelles. L'ensemble des auteurs s'accorde dans l'ensemble à abandonner une forme de récit national, artificiel dans ses continuités, oublieux des minorités, et par conséquent jugé intenable. Deux perspectives sont présentées comme mettant à distance, voire en crise, un récit national focalisé sur l'émergence de l'État et de la nation : une perspective d'ouverture de l'histoire ukrainienne aux phénomènes globaux et transnationaux, et une perspective mettant l'accent sur les disparités régionales.

L'approche transnationale vise à faire en sorte que l'histoire de l'Ukraine prenne pleinement sa place dans l'histoire européenne et en retour à être éclairée par elle. C'est notamment ce prisme ukrainien de l'histoire européenne que défend Andrea Graziosi dans sa contribution. Une perspective notamment adoptée par George Liber et Mark von Hagen pour envisager l'enchâssement des formes de violence du $\mathrm{XX}^{\mathrm{e}}$ siècle européen sur un territoire qui en a vu se succéder de nombreuses. M. von Hagen applique notamment une lecture héritée de l'« imperial turn » afin de souligner tout à la fois le dynamisme et les innovations dans les violences d'occupation amorcées avec la Première Guerre mondiale ${ }^{3}$. Si la catégorie coloniale est suggestive, l'article ne propose ensuite malheureusement que des tableaux successifs des différents régimes d'occupation. Ce désenclavement de l'Ukraine est aussi favorisé par l'accent mis sur l'histoire culturelle, intellectuelle et artistique, permettant de souligner combien une histoire strictement ukrainienne serait pauvre, mais combien présenter l'Ukraine comme un carrefour où se nouent et s'élaborent des influences multiples permet tout à la fois d'envisager la particularité de ce territoire sans le replier sur lui-même. Dans la lignée de ses travaux récents sur les milieux théâtraux et culturels ${ }^{4}$, Mayhill Fowler distingue ainsi clairement la « culture ukrainienne » de la « culture en Ukraine », au profit de la seconde, ouvrant de nombreuses pistes. 
Presque en symétrique, une série de contributions met l'accent sur les variations régionales dans l'histoire ukrainienne. La Galicie est évoquée - et attendue - mais les études de cas font varier la focale, tantôt plus large ou plus courte. Les textes de Faith Hillis et Heather Coleman illustrent la tension entre particularismes locaux et élaborations de projets politiques intégrés à l'empire tsariste. L'article de Serhii Plokhy étudie les vertus d'une comparaison interne dans le cadre d'une étude de la grande famine de 1932-1933. Présentant par ailleurs de nombreuses cartes (claires et en couleur), S. Plokhy montre les nombreuses variations régionales (pertes, mortalité, production, répressions, aides) dans la catastrophe subie par les campagnes ukrainiennes.

Les contributions portant sur l'enseignement, l'ancrage social et médiatique de l'histoire, en Ukraine mais aussi en Russie et en Amérique du Nord se fondent sur des éléments préalables aux événements liés à Maïdan, à l'annexion de la Crimée et à la guerre à l'Est de l'Ukraine. Si plusieurs articles ouvrent des pistes sur cet après, ils donnent pour l'ensemble l'impression de décrire une période révolue, où l'histoire ukrainienne était notamment tiraillée entre passé soviétique, monde russe et récit national. Les cartes ont été depuis rebattues, mais les articles ne constituent pas moins des contributions précieuses sur ces tensions mémorielles et l'ambivalence des mots et des catégories employés. En creux, ils n'en soulignent que mieux les rapides évolutions récentes.

Un volume de synthèse, aussi conséquent soit-il, ne peut traiter de tous les sujets. On regrettera néanmoins que certains objets de recherche, parfois polémiques, souvent douloureux, ne soient pas abordés. Certains champs - généralement traités par des chercheurs extérieurs aux Ukrainian Studies à proprement parler, et intégrés aux études en histoire soviétique, de l'Europe centrale et orientale, ou en histoire juive voire de la Shoah - forment quelques angles morts. Le dynamisme des recherches que trahit ce volume fournira malgré tout une utile introduction aux étudiants soucieux de synthèses historiographiques. Il permettra aussi aux chercheurs distraits en histoire de l'Europe centrale et orientale, parfois trop centrés sur la Russie, de se mettre aisément à jour. Dans l'espoir aussi qu'il suscitera l'intérêt de chercheurs qui n'auraient pas imaginé se pencher sur ces régions du monde.

1 - George O. Liber, Total Wars and the Making of Modern Ukraine, 1914-1954, Toronto : University of Toronto Press, 2016 ; Faith Hillis, Children of Rus : Right Bank Ukraine and the Invention of a Russian Nation, Ithaca : Cornell University Press, 2013.

2 - Mark von Hagen, « Does Ukraine Have a History? », Slavic Review, 54, 3 (Automne 1995), p. 658-673.

3 -Approche développée depuis, non sans soulever quelques problèmes, par Stephen Velychenko, Painting Imperialism and Nationalism Red : The Ukrainian Marxist Critique of Russian Communist Rule in Ukraine, 1918-1925, Toronto : University of Toronto Press, 2015. 4 - Mayhill C. Fowler, Beau Monde on Empire's Edge: State and Stage in Soviet Ukraine, Toronto : University of Toronto Press, 2017.

\section{Thomas Chopard}

